\title{
Crew Exploration Vehicle Ascent Abort Overview
}

\author{
John Davidson ${ }^{*}$ \\ NASA Langley Research Center, Hampton, VA 23681 \\ Jennifer Madsen ${ }^{\dagger}$, Ryan Proud ${ }^{\ddagger}$, and Deborah Merritt ${ }^{\dagger}$ \\ NASA Johnson Space Center, Houston, TX 77058 \\ David Raney ${ }^{\S}$ and Dean Sparks ${ }^{\S}$ \\ NASA Langley Research Center, Hampton, VA 23681 \\ Paul Kenyon ${ }^{* *}$ and Richard Burt ${ }^{\dagger \dagger}$ \\ Lockheed Martin Corporation, Denver, CO 80201 \\ Mike McFarland ${ }^{+}$ \\ Orbital Sciences Corporation, Dulles, VA 20166
}

\begin{abstract}
One of the primary design drivers for NASA's Crew Exploration Vehicle (CEV) is to ensure crew safety. Aborts during the critical ascent flight phase require the design and operation of CEV systems to escape from the Crew Launch Vehicle and return the crew safely to the Earth. To accomplish this requirement of continuous abort coverage, CEV ascent abort modes are being designed and analyzed to accommodate the velocity, altitude, atmospheric, and vehicle configuration changes that occur during ascent. The analysis involves an evaluation of the feasibility and survivability of each abort mode and an assessment of the abort mode coverage. These studies and design trades are being conducted so that more informed decisions can be made regarding the vehicle abort requirements, design, and operation. This paper presents an overview of the CEV, driving requirements for abort scenarios, and an overview of current ascent abort modes. Example analysis results are then discussed. Finally, future areas for abort analysis are addressed.
\end{abstract}

\section{Nomenclature}

Acronyms:

$\begin{array}{ll}\text { ACM } & \text { Attitude Control Motor } \\ \text { ADL } & \text { Abort Decision Logic } \\ \text { AGL } & \text { Above Ground Level } \\ \text { AOA } & \text { Abort Once Around } \\ \text { ATO } & \text { Abort To Orbit } \\ \text { BPC } & \text { Boost Protective Cover } \\ \text { CEV } & \text { Crew Exploration Vehicle } \\ \text { CLV } & \text { Crew Launch Vehicle } \\ \text { CM } & \text { Crew Module } \\ \text { DAEZ } & \text { Down-range Abort Exclusion Zone } \\ \text { GRAM } & \text { Global Reference Atmospheric Model }\end{array}$

\footnotetext{
*Aerospace Engineer, Dynamic Systems and Control Branch, M/S 308, Senior Member AIAA.

'Aerospace Engineer, Integrated GN\&C Analysis Branch.

* Aerospace Engineer, GN\&C Autonomous Flight Systems Branch, Member AIAA.

$\S$ Aerospace Engineer, Dynamic Systems and Control Branch.

${ }^{* *}$ Embedded S/W Engineer Sr. Stf., Space System Company.

${ }^{\dagger}$ System Engineer Sr., Space System Company.

$\$$ Scientist, Space Systems Group, AIAA Associate Felllow.
} 


$\begin{array}{ll}\text { ISS } & \text { International Space Station } \\ \text { KSC } & \text { Kennedy Space Center } \\ \text { LAS } & \text { Launch Abort System } \\ \text { LAV } & \text { Launch Abort Vehicle (LAS with CM) } \\ \text { LES } & \text { Launch Escape System (Apollo) } \\ \text { MET } & \text { Mission Elasped Time } \\ \text { OME } & \text { Orion Main Engine } \\ \text { RCS } & \text { Reaction Control System } \\ \text { SA } & \text { Spacecraft Adapter } \\ \text { SM } & \text { Service Module } \\ \text { RCS } & \text { Reaction Control System } \\ \text { RTAL } & \text { Retrograde Targeted Abort Landing } \\ \text { TAL } & \text { Targeted Abort Landing } \\ \text { UAS } & \text { Untargeted Abort Splashdown }\end{array}$

\section{Introduction}

NASA's Vision for Space Exploration calls for a long term exploration of the Moon, Mars, and beyond, with a focus on returning astronauts to the Moon by 2020. These goals require the development of a new spacecraft - the Crew Exploration Vehicle (CEV) [1]. One of the primary design drivers for the Crew Exploration Vehicle is to ensure crew safety. CEV requirements specify that abort capability must be continuously available from the launch pad until the mission destination is reached [2]. Aborts during the critical ascent flight phase require the design and operation of CEV systems to escape from the Crew Launch Vehicle (CLV) and return the crew safely to the Earth.

Ascent phase aborts are characterized by large changes in vehicle altitude, large amplitude attitude maneuvers, and large vehicle center-of-gravity movement; and pose significant engineering challenges. Several ascent abort modes are being designed and analyzed to accommodate the velocity, altitude, atmospheric, and vehicle configuration changes that occur during ascent. These modes provide abort coverage extending from the launch pad until the CEV achieves a sustainable orbit. Analyzing these modes involves an evaluation of the feasibility and survivability of each abort mode and an assessment of the abort mode coverage using the current baseline vehicle design. Factors such as abort system performance, concept of operations, crew load limits, thermal environments, crew recovery, and vehicle element disposal are investigated to determine if the current vehicle requirements are appropriate and achievable.

This paper presents an overview of the CEV in Section 2, driving requirements for abort scenarios in Section 3, and an overview of ascent abort modes in Section 4. Example analysis results are then discussed in Section 5. Finally, future areas for abort analysis are addressed in Section 6.

\section{Crew Exploration Vehicle}

The Crew Exploration Vehicle (CEV) is based on a design similar to the Apollo program's Command and Service Modules and is composed of four main elements: the Launch Abort System, the Crew Module, the Service Module, and the Spacecraft Adapter as shown in Figure 1 [3]. The Launch Abort System (LAS) provides a reliable abort capability for aborts that occur within the atmosphere. The Crew Module (CM) provides a safe habitable volume for the crew during launch, spaceflight, and return from the International Space Station (ISS) or Moon. The Service Module (SM) provides additional resources necessary to support the primary mission, including power and maneuvering capability. The SM is also used to provide abort capability for exo-atmospheric aborts. The Spacecraft Adapter (SA) provides the interface between the spacecraft and the Crew Launch Vehicle (CLV). The term Launch Abort Vehicle (LAV) will be used to refer to the LAS and CM together, prior to jettison of the LAS.

As shown in Figure 2, the LAS consists of an abort motor to provide the CLV to LAV abort separation function, an Attitude Control Motor (ACM) to provide attitude and rate control, a pair of deployable canards to passively assist in orienting the CM to heat-shield forward, and a jettison motor for CM to LAS separation. The ACM consists of a throttle-able solid rocket system. The LAS also provides a Boost Protective Cover (BPC) that shields the CM from debris and the aero-thermal environment during ascent.

In addition to its nominal mission functions, the SM also provides abort capability during the high altitude portions of the CLV launch profile. The SM contains two propulsion systems: the Service Module Orion Main Engine (SM OME) (used for large maneuvers, such as orbit changes and re-entry) and the Reaction Control System 
(RCS) (used for fine control, such as proximity operations and docking, as well as providing a backup for the OME). For LAS assisted aborts, the LAV separates from the SM at the SM-CM plane. In the event of a CLV problem after nominal jettison of the LAS, the SM OME and RCS thrusters can be fired to maneuver the CEV to safety.

\section{CEV Ascent Abort Requirements}

The driving requirements for CEV ascent aborts [2] are as follows:

- The CEV shall perform aborts from the time the CEV abort system is armed on the launch pad until the mission destination is reached.

- The CEV shall automatically determine the need for an abort.

- The CEV shall automatically initiate the ascent abort sequence.

- The CEV shall automatically perform abort maneuvers.

- The CEV shall provide sufficient range and altitude performance during pad and low altitude aborts to achieve a water landing, with full Landing and Recovery System (LRS) deployment and functionality, for 95\% (final requirement To Be Resolved (TBR)) of the pad abort wind cases as defined in the Design Specification for Natural Environments (DSNE) [4] and thermal radiation environments as defined in the (requirement To Be Determined (TBD)), such that CEV landing ensures no greater than low risk of crew injury as defined by the Human-Systems Integration Requirements (HSIR) [5].

- The CEV shall provide abort thrust to achieve positive separation for all abort conditions.

- The CEV shall automatically perform targeting and maneuvers to depart from the CLV.

- The CEV shall automatically perform targeting and maneuvers for abort to orbit.

- The CEV shall provide ascent aborts for ISS missions that result in landing outside the Down-range Abort Exclusion Zone (DAEZ). (The DAEZ is a region in the Atlantic Ocean along a nominal CLV ascent trajectory to be avoided for CEV landings following launch aborts for ISS and Lunar crewed missions.)

- The CEV shall abort without relying on thrust from the CLV.

Additional derived Guidance, Navigation, and Control (GN\&C) subsystem specifications [6] have been established to assess CEV abort performance as follows:

- Altitude and downrange requirements for pad abort and near pad abort to ensure LAV abort trajectories will result in a water landing to increase crew survivability, and achieve sufficient altitude to ensure safe deployment of the Landing and Recovery System.

- For pad and near pad aborts, the LAV shall achieve a minimum distance from the pad at LAS jettison from the Crew Module.

- For pad and near pad aborts, the Crew Module body attitudes and rates at LAS jettison shall be within specified limits to allow successful Landing and Recovery System deployment.

Detailed descriptions and design driving analysis are described in References [2] and [3].

\section{CEV Ascent Abort Mode Overview}

The CEV system is required to provide a launch abort capability from the launch pad after CM hatch closure throughout the CLV ascent until orbital insertion. The possible reasons for an ascent abort fall into one of three general categories: (1) a partial or total loss of CLV propulsion, (2) a loss of CLV control, or (3) a systems failure, either on the CLV or CEV, which results in the inability to safely achieve orbit. Several abort modes are required to accommodate the velocity, altitude, atmospheric, and vehicle configuration changes that occur during ascent. These modes provide abort coverage extending from the launch pad until the CEV achieves a sustainable orbit.

Mode I aborts, also referred to as Launch Abort System (LAS) aborts, are performed using the LAS and remain a viable option until the LAS is nominally jettisoned early in second stage. During Mode I aborts, the LAS abort motor is used to pull the Crew Module (CM) away from the CLV and Service Module (SM). Mode I aborts are characterized by high aerodynamic loads induced by low altitude maneuvers and high accelerations caused by the launch abort motor. LAS aborts may be commanded via the ground-based health management system, the on-board CEV Abort Decision Logic (ADL), the crew, or ground personnel.

Mode II aborts, also referred to as Untargeted Abort Splashdown (UAS) aborts, do not utilize the LAS. Instead, the CLV upper stage engine is shut down and the SM Reaction Control System (RCS) is used to provide adequate 
clearance between the launch vehicle and CEV. Once the CEV is sufficiently far away from the launch vehicle, the CM separates from the SM, and then the CM is maneuvered for a guided re-entry, and descends using parachutes to a safe landing location.

Mode III aborts, commonly known as Targeted Abort Landings (TAL), are triggered by late second stage failures where the CEV trajectory is modified via a targeted SM OME burn followed by a CM guided entry to a target landing site. The goal of these trajectory control efforts is to select a landing site that maximizes the chances of crew survival and recovery, while also meeting crew loads and SM thermal constraints. Due to the thermal environment associated with "drooping" into the atmosphere following an upper stage engine failure, the focus of the Mode III analysis is the droop altitude for various scenarios and the ways in which the droop effect can be minimized.

The last type of abort mode is Mode IV, which is an Abort To Orbit (ATO). This mode describes cases where an abort is performed following a premature shutdown of the upper stage when the SM has sufficient capability to achieve a safe orbit insertion and de-orbit burn. Similar to the TAL aborts, the ATO cases must protect the SM from adverse thermal conditions. For the Mode IV abort, the analysis looks at the amount of propellant (or $\Delta \mathrm{V}$ ) required to achieve a sustainable orbit, and similar to TAL, focuses primarily on the droop altitude effects.

\section{A. Launch Abort System Aborts}

Launch Abort System (LAS) aborts, or Mode I aborts, are performed using the LAS and remain a viable option until the LAS is nominally jettisoned at a pre-designated point during the flight of the CLV second stage. The current CEV design uses an active LAS approach that stabilizes and reorients the capsule during the launch abort maneuver using an Attitude Control Motor (ACM) in the escape tower as shown in Figure 3. This design also includes "Apollo-like" deployable passive canards to assist the ACM during the reorientation maneuver. This approach differs from the passive approach used on Apollo which relied upon the use of open-loop timers to control the abort event sequencing, along with a passive canard that was deployed to reorient the vehicle to a heat-shield forward attitude following the escape motor firing and a brief coast period. The Apollo Launch Escape System (LES) [7] is shown in Figure 4. The passive Apollo system required approximately $1000 \mathrm{lb}$ of ballast to ensure aerodynamic stability during the escape motor firing and coast, and the passive canard system could produce tumbling in some portions of the abort envelope before settling into a heat-shield forward attitude.

The use of the active LAS on the CEV enables elimination of the ballast that would otherwise be required to ensure passive aerodynamic stability in the tower nose forward orientation, as well as the elimination of the openloop pitch motor weight that was also included in the passive Apollo. A feedback control algorithm is currently under development that issues commands to the ACM system to stabilize and reorient the LAV during the abort. The current concept controller uses a proportional/integral/derivative (PID) approach to control attitudes and rates of the LAV.

The concept of operation and event sequencing during an abort varies with altitude as described in Figure 5. The LAS aborts are divided into three regimes by altitude above ground level (AGL) at abort initiation as follows: Low: 0 to $25 \mathrm{k}$ feet; Mid: $25 \mathrm{k}$ feet to $150 \mathrm{k}$ feet; High: $150 \mathrm{k}$ feet (ft) to nominal LAS jettison. The sequence of events during an abort is based on abort initiation altitude, time elapsed from abort initiation, and on estimated dynamic pressure and Mach.

Once an abort condition is detected, the ADL ignites the abort motor and ACM. After the abort motor burns out (approximately 4 seconds), the vehicle continues on a controlled coast trajectory. The ACM provides the control during abort motor operation and coast to maintain nose-forward flight. The reorientation maneuver is initiated at an elapsed time between 8 and 15 seconds to orient the LAV with the heat-shield into the velocity vector, assuring proper attitude for parachute deployment and, from high altitudes, proper thermal protection system (TPS) orientation. The ACM is used to track the reorientation maneuver command. At the start of the reorientation, the canards are deployed (to a fixed open position) to assist the reorientation. The deployed canards also effectively eliminate possible nose-forward trim conditions, ensuring that the LAV can not inadvertently tumble back into the nose forward attitude. Following reorientation, the ACM is used to stabilize the LAV until the LAS is jettisoned from the CM.

There is a downrange and altitude requirement that the trajectory must achieve at LAS jettison to ensure a successful chute deployment and water landing following a pad abort. To achieve these requirements, a pitch maneuver must be performed during the abort motor burn, so that the abort motor thrust is directed along the proper trajectory. The brevity of the abort motor firing period means that the pitch maneuver must be fairly abrupt if it is to be effective in directing the abort trajectory. The high rates and attitudes of the pitch maneuver in the pad abort regime make it one of the most demanding of all the abort cases in terms of the authority required from the ACM. 
Sufficient control authority must be provided to ensure that the LAV does not tumble due to abort motor thrust misalignments or aerodynamic uncertainties during this portion of the abort.

\section{B. Service Module Aborts}

This section provides an overview of the Service Module abort modes. Service Module (SM) aborts are characterized by using the SM propulsion systems to safely maneuver the CEV away from a failed CLV during the second stage of ascent following nominal LAS jettison. SM abort coverage is obtained through the capability to complete an Untargeted Abort Splashdown (UAS), Retrograde Targeted Abort Landing (RTAL), Targeted Abort Landing (TAL), Abort to Orbit (ATO), or Abort Once Around (AOA).

\section{Untargeted Abort Splashdown (UAS)}

Untargeted Abort Splashdown (UAS), or Mode II aborts, are the result of upper stage CLV engine failure after the nominal jettison of the LAS and prior to reaching sufficient velocity to use the SM Orion Main Engine (OME) to propel the CEV to the far side of the Atlantic Ocean. A UAS abort is the primary abort mode over the largest portion of the ascent trajectory, from approximately 160 seconds mission elapsed time (MET) to approximately 550 seconds MET.

In order to achieve a successful UAS abort, the CM must be flying by itself in a heat-shield forward attitude prior to falling through an altitude of $300,000 \mathrm{ft}$. A primary constraint on this goal is that the $\mathrm{CM}$ can not land in the water more than 150 nautical miles (nmi) to the East of St. John's airport in Canada. In order to get into the proper attitude after a CLV failure, the CEV must: 1) separate from the CLV using SM RCS jets after the CLV thrust has decreased to nearly zero, 2) jettison the SM in the proper attitude, 3) jettison the docking mechanism in the proper attitude, and 4) reorient the CM into a heat-shield forward attitude. Analysis has shown that this sequence can be performed in less than 90 seconds. Relative separation margins are imparted after separation by the SM RCS jets and docking mechanism jettison spring, respectively.

At $300,000 \mathrm{ft}$ the aerodynamic forces begin to build up and the CM controls its lift vector to satisfy the $150 \mathrm{nmi}$ out to sea constraint while trying to minimize loads on the crew. Parachute deployment occurs at the standard endof-mission altitude, around 50,000 ft. Figure 6 (from Reference [8]) shows an altitude versus downrange distance trajectory from the initiation of a UAS abort. The dotted line illustrates how the downrange capability of the CEV on a UAS abort is very limited due to the lack of additional thrust.

\section{2. $\quad$ Targeted Abort Landing (TAL)}

Mode III aborts include both Retrograde Targeted Abort Landings (RTAL) and Targeted Abort Landings (TAL). RTAL aborts are necessary during a small ascent time region between UAS and TAL. This is approximately between 550 seconds and 555 seconds MET. During this portion of the nominal ascent, there is insufficient energy and SM propellant to use the OME thrust to reach a TAL site, but too much energy to land within 150nmi of the St. John's, Canada, using entry lift control alone.

In order to achieve a successful RTAL, a short SM OME burn is performed to decrease horizontal velocity, lower the flight path angle, and keep the CM within $150 \mathrm{nmi}$ of St. John's. A primary constraint on this SM OME burn is that the burn must finish with enough time available for the CM and SM to separate, the CM and docking mechanism to separate, and the CM to achieve heat-shield forward attitude prior to reaching an altitude of 300,000 ft. Figure 7 shows an altitude versus downrange distance trajectory from the initiation of an RTAL abort. For this type of abort, the altitude loss above $400,000 \mathrm{ft}$ is higher than with a UAS abort due to the SM OME firing in a rearward and downward direction.

This is a relatively new abort mode option for CEV, but retrograde abort burns have been considered before. Apollo included a retrograde burn option to target a ship in the North Atlantic Ocean for engine failures late in ascent. The Space Shuttle includes a retrograde burn option for early engine failures to return to a safe landing in Florida.

Targeted Abort Landing (TAL) aborts require firing the SM OME to impart additional velocity to the spacecraft in order to achieve landing near the TAL sites. The TAL sites include Shannon, Ireland and Cape Verde, Africa for ISS and Lunar missions, respectively. The TAL abort boundary begins the first time that the CM can get within a circle $150 \mathrm{nmi}$ west of the TAL site and ends when either the abort dynamics show that the SM disposal is impinging on land or an Abort To Orbit (ATO) option is achieved. This is approximately between 555 seconds and 570 seconds MET.

In order to achieve a successful TAL abort, the CM must land in the water within $150 \mathrm{nmi}$ of recovery forces. The primary constraint for this abort mode is a thermal altitude limit that restricts firing the SM below 400,000 ft. Propellant is not a constraint for TAL because the propellant load for the mission is sized either to maximize TAL capability on ISS missions or to achieve a much larger Trans-Lunar Injection burn on Lunar missions with plenty of 
excess propellant for aborts. Following CLV separation using the SM RCS jets, the SM OME burns in a relatively high local-vertical-local-horizontal (LVLH) pitch attitude burn (60-70 degrees) to arrest the negative altitude rate and avoid the thermal minimum altitude constraint. Figure 8 (from Reference [8]) shows an altitude versus downrange distance trajectory plot from TAL abort initiation and continuing until CM reentry and landing. The positive altitude rate that occurs while the SM OME is firing above 400,000 ft is necessary to increase the vehicle's downrange distance capability and to reach the target landing site.

\section{3. $\quad$ Abort To Orbit (ATO)}

Abort To Orbit (ATO), or Mode IV aborts, result when a CLV Upper Stage engine failure occurs late enough that the SM has sufficient thrust and propellant to achieve sustainable orbit. ATO is the primary abort mode from approximately 570 seconds MET to nominal shutdown of the upper stage at approximately 590 seconds MET.

In order to achieve a successful ATO abort, two SM OME burns are performed after CLV separation. The first burn raises orbital apogee to $100 \mathrm{nmi}$. The second burn circularizes the orbit. The ATO is considered complete upon circularization as nominal orbit operations are performed prior to a nominal deorbit burn. Figure 9 (from Reference [8]) shows an altitude versus downrange distance trajectory plot that shows the altitude gain that is generated by the SM OME burn after ATO abort initiation. This altitude will continue to climb to the target apogee of $100 \mathrm{nmi}$ before a second circularizing burn will be needed.

Abort Once Around (AOA) is a potential down-mode abort capability if performing a TAL or ATO abort fails and enough performance is available in the system. The abort is characterized by performing 1,2 or 3 OME burns to land in or near the contiguous United States (CONUS) or Mexico. These aborts would typically be performed for systems failures during ascent that required immediate return of the crew to Earth. An AOA could also be performed after the nominal insertion burn if, for example, the Crew Module solar arrays failed to deploy.

Figures 10-13 (from Reference [9]) show latitude versus longitude ground tracks for potential AOA aborts from either ISS or Lunar missions. For these figures, land landings were evaluated to White Sands (WS), Yuma (YMA), Edwards (EDW), and Tonopah, NV (TNP). For the close-up versions in Figures 11 and 13, SM landings are shown as heel and toe objects, depending on assumed ballistic numbers after separation. For ISS missions only, White Sands is a possibility due to propulsion limitations. For Lunar missions, the other sites become available.

\section{Ascent Abort Performance Analysis}

Ascent abort performance analysis involves an evaluation of the feasibility and survivability of each abort mode and an assessment of the abort mode performance and CLV trajectory coverage using the current baseline vehicle design. Factors such as abort system performance, concept of operations, crew load limits, thermal environments, crew recovery, and vehicle element disposal are being investigated to determine if the current vehicle requirements are appropriate and achievable. These studies and design trades are being conducted so that more informed decisions can be made regarding the vehicle requirements, design, and operation. Examples of two types of analysis being used to assess abort performance are: ascent abort trajectory surveys and dispersed parameter (Monte Carlo) analysis. LAS aborts will be used as examples to describe analysis studies, but the SM aborts are analyzed using a similar process.

\section{A. Ascent Abort Trajectory Surveys}

Ascent Abort Trajectory Surveys consist of initiating abort simulations at n-second intervals along a nominal CLV ISS ascent trajectory. From the launch pad to an altitude of 300,000 ft, the active LAS system provides abort coverage. Simulations are run with the nominal (non-dispersed) model, and with zero imparted angular rates and attitudes (i.e. no CLV attitude control failure) at abort initiation. This set of simulated aborts is used to verify that the controller gains and event sequencing parameters are appropriately scheduled to provide acceptable performance in all flight regimes. Example time histories from an ascent abort trajectory survey (from Reference [10]) are shown in Figure 14. For this survey, aborts were initiated at 1-second intervals along the CLV trajectory. In this figure, the time histories are color-coded by altitude at abort initiation per the annotation in the figure. The top portion of the figure shows angle-of-attack plotted against time from abort initiation. For this analysis, the angle-of-attack, as illustrated in Figure 15, is defined as 0 degrees when the LAS nose (top of LAS tower) points forward directly into the freestream, while 180 degrees denotes the heat-shield forward orientation. The angle-of-attack variation during the first 5 seconds of the low altitude aborts indicates the initial pitch maneuver that is performed to direct the abort trajectory away from the launch pad and out over the water. The maneuver to reorient the LAV from a nose-forward to a heat-shield forward attitude is also apparent in the angle-of-attack time histories. The lower portion shows 
sideslip angle plotted against time since abort initiation. The initial sideslip angle variations in the pad abort regime are due to the initial pitch maneuver and then the larger variations are due to the reorientation maneuver.

\section{B. Dispersed Parameter (Monte Carlo) Analysis}

To help assessment of ascent abort performance subject to potential uncertainties and possible variations in the system parameters, a series of Monte Carlo analyses are being conducted. The perturbations in these Monte Carlo runs include the following system parameters for LAS abort studies: 1) LAS abort motor mis-alignment and thrust level output (due to temperature variation); 2) LAS ACM and abort motor points of action; 3) aerodynamics data; and 4) LAS and CEV mass properties (i.e., mass, inertia and center of mass locations). Also, variations in atmospheric properties were provided by the Global Reference Atmospheric Model (GRAM-99) [11].

In addition to the system parameter dispersions, variations in initial flight conditions (for CEV abort initiation) are also considered. These dispersion values are taken from separate Monte Carlo runs of the CLV booster simulation, with variations in the CLV model parameters. From these CLV Monte Carlo runs, flight conditions for critical abort points along the CLV trajectory (e.g., pad abort, maximum dynamic pressure, and abort at high altitude) are obtained for use in the CEV Monte Carlo analysis set.

Figure 16 (from Reference [10]) shows example Monte Carlo analysis of the reorientation maneuver (maneuver from nose-forward to heat-shield forward flight prior to LAS tower jettison) for the pad abort scenario. The top portion of the figure shows angle-of-attack plotted against time from abort initiation. The angle-of-attack is defined as 0 degrees when the LAS nose points forward directly into the freestream, while 180 degrees denotes the heat-shield forward orientation. The lower portion shows sideslip angle plotted against time since abort initiation. This time histories show that given system parameter dispersions, the LAV successfully maneuvers from a noseforward to a heat-shield forward attitude with acceptable levels of sideslip angle.

\section{Concluding Remarks}

This paper has presented an overview of the Crew Exploration Vehicle (CEV), driving requirements for ascent abort scenarios, and an overview of ascent abort modes. One of the primary design drivers for the CEV is to ensure crew safety. CEV requirements specify that abort capability must be continuously available from the launch pad until the mission destination is reached. Aborts during the critical ascent flight phase require the design and operation of CEV systems to escape from the Crew Launch Vehicle (CLV) and return the crew safely to the Earth. Several ascent abort modes have been designed and analyzed to accommodate the velocity, altitude, atmospheric, and vehicle configuration changes that occur during ascent. These modes provide abort coverage extending from the launch pad until the CEV achieves a sustainable orbit.

The baseline CEV has been tested with the Government and Contractor simulation tools to evaluate abort performance and capability to meet the current CEV abort performance requirements, from launch on a typical mission to the ISS. Critical abort scenarios have been identified and are being analyzed using nominal vehicle (abort trajectory surveys) and dispersed system parameter (Monte Carlo) simulation analysis tools.

Current and future areas for abort analysis includes:

- On-going analyses of abort performance and requirement compliance as future aerodynamic and vehicle design updates become available.

- Designing and analyzing LAV guidance algorithms which use the vehicle lift capabilities to achieve improved low altitude downrange and water landing performance.

- Attitude Control Motor (ACM) Jet Interaction, defined as the attitude control attenuating characteristic caused by the ACM plume interaction with the aerodynamic flow around the vehicle, has been identified as a concern for control and performance of the LAV. Analysis and testing is planned to quantify the magnitude of this effect, assess any performance impact, and develop viable design modifications to mitigate any deleterious effects.

- Preliminary structural analysis is predicting the first free-free bending mode of the LAV to be closer to the controller cross-over frequency than typical industry standards would allow. Additional analysis and testing is planned to better quantify the structural bending mode frequencies and to assess LAV controller filtering and bandwidth modification options.

- Analysis to assess an updated LAS design which achieves lower aerodynamic drag to improve both performance to orbit and LAV abort performance. 
- Guidance, Navigation, and Control algorithm refinement is necessary for the Mode III and Mode IV SM aborts. Improvements over the current algorithms may be necessary to ensure abort coverage for performance degrading effects like non-optimal launch times and dispersed CLV performance.

- Both LAS and SM abort modes will drive CEV weight due to structural loads and thermal effects. Analysis will be performed to determine if more benign abort modes could be implemented which have lower weight impacts.

\section{References}

1. "Exploration Systems Architecture Study Final Report," NASA TM-2005-214062, November 2005.

2. "Crew Exploration Vehicle System Requirements Document," NASA CEV Document Number: CxP72000, January 2007.

3. "Crew Exploration Vehicle Architecture Design Document," NASA CEV Document Number: CxP-72140, January 2007.

4. "Constellation Program Design Specification for Natural Environments (DSNE)," NASA Document Number: CxP-70023, January 2007.

5. "Constellation Program Human-Systems Integration Requirements (HSIR)," NASA Document Number: CxP-70024, January 2007.

6. "Crew Exploration Vehicle Subsystem Requirements Specification Guidance, Navigation, \& Control." NASA Document CEV-T-031210, July 2007.

7. McCarthy, J. F. JR., Dodds, J. Ian, Crowders, R. S., "Development of the Apollo Launch Escape System," J. Spacecraft, Vol. 5, No. 8, p.927, North American Rockwell Corporation, Downey, CA, Aug. 1968.

8. Falck, R. D. and Gefert, L. P., "Crew Exploration Vehicle Ascent Abort Trajectory Analysis and Optimization", AIAA Guidance, Navigation and Control Conference, Hilton Head, SC, August 2007.

9. Shoemaker, et al. "Service Module Abort Coverage Analysis", NASA Document Number CEV-T-009, June 2007.

10. Sparks, D. and Raney, D., "Crew Exploration Vehicle Launch Abort System Controller Performance Analysis," AIAA Guidance, Navigation, and Control Conference, Hilton Head, SC, August 2007.

11. Justus, C. G., Johnson, D. L., The NASA/MSFC Global Reference Atmospheric Model -1999 Version (GRAM-99), NASA-TM-1999-209630, May 1999.

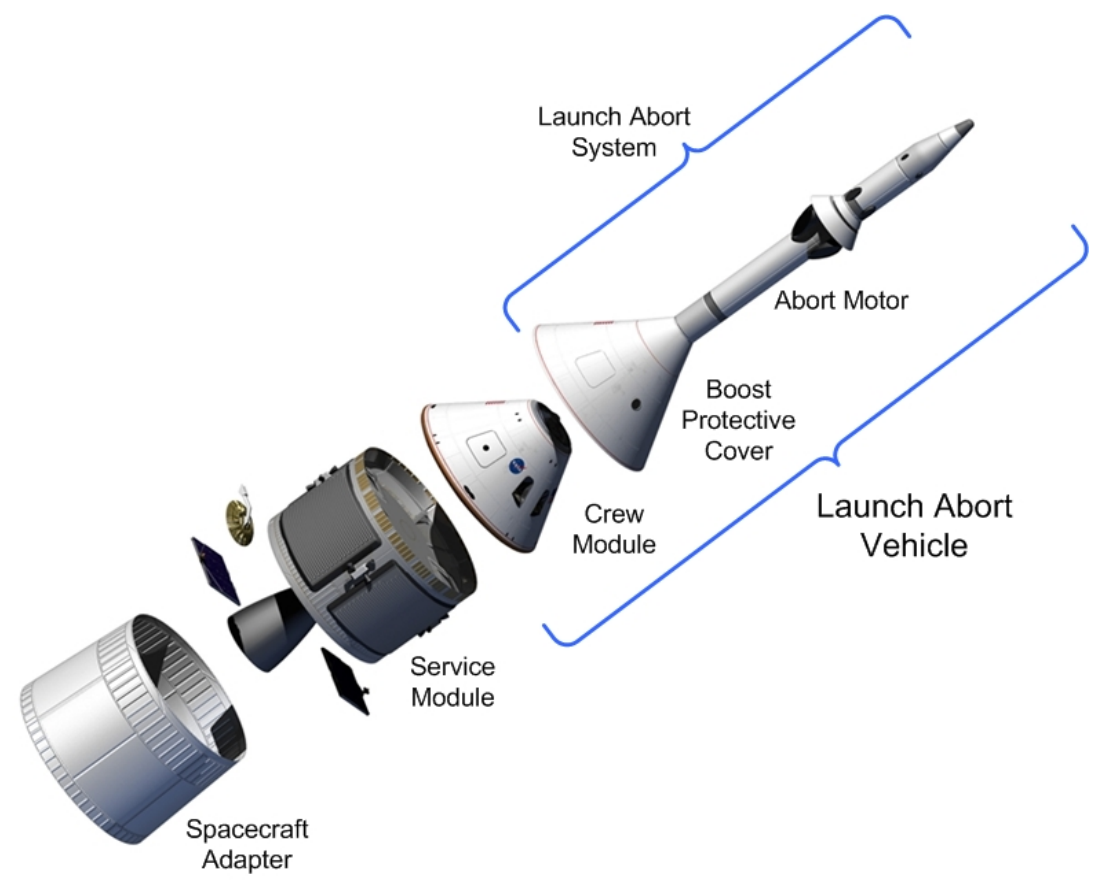

Figure 1. Crew Exploration Vehicle. 


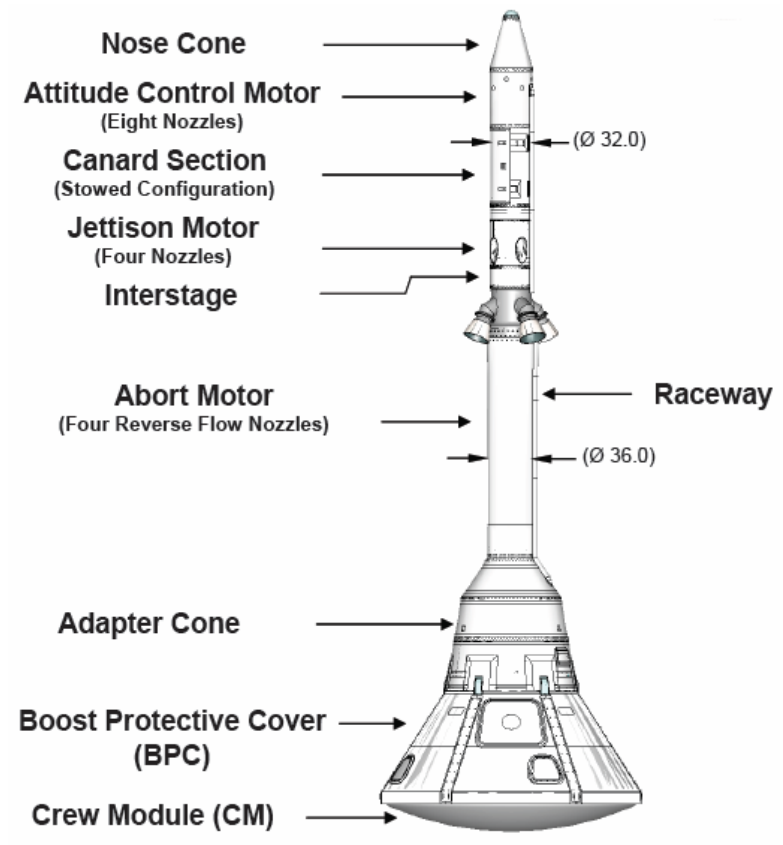

Figure 2. Crew Exploration Vehicle Launch Abort Vehicle.

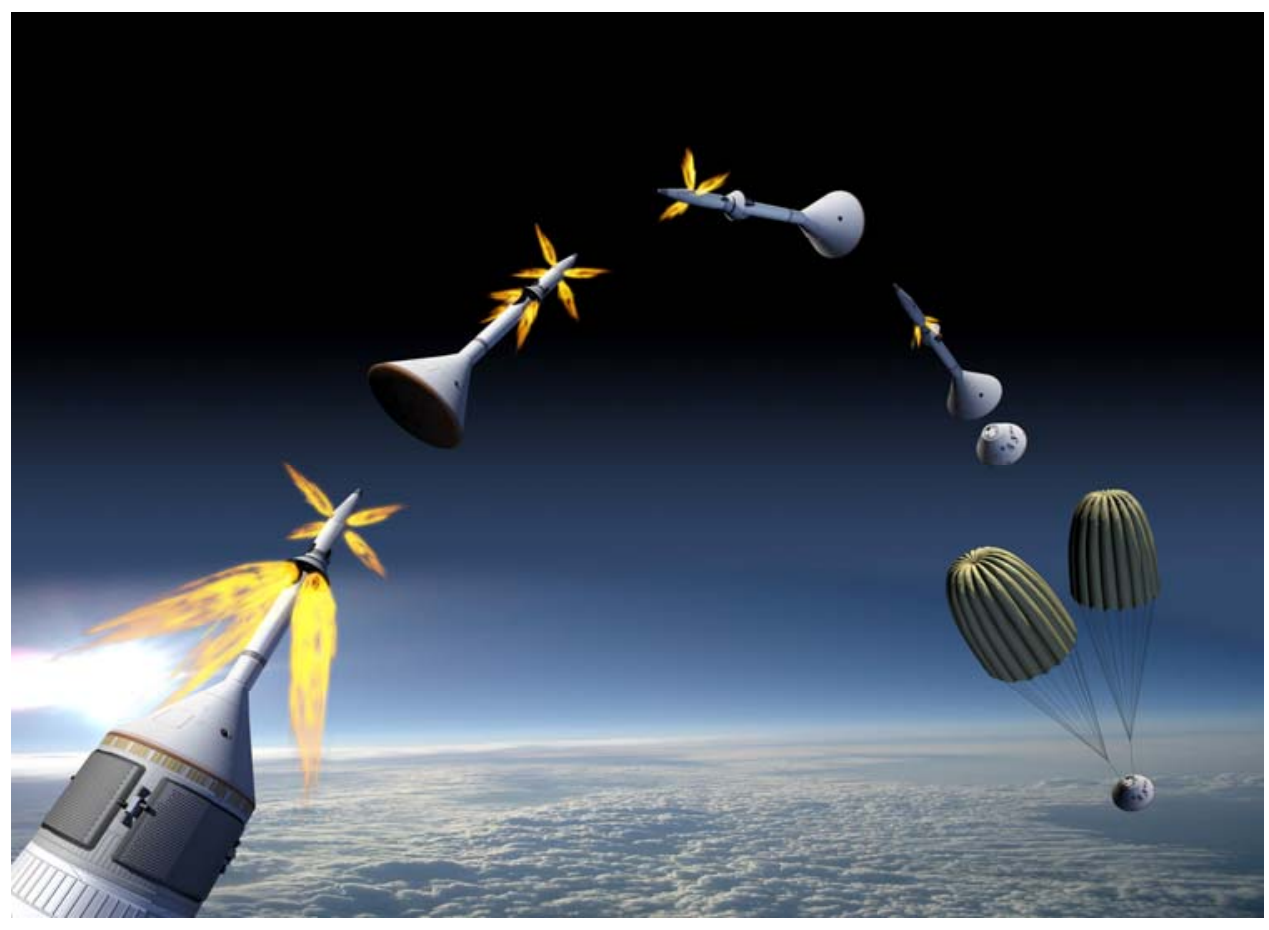

Figure 3. CEV Launch Abort System Abort Scenario. 


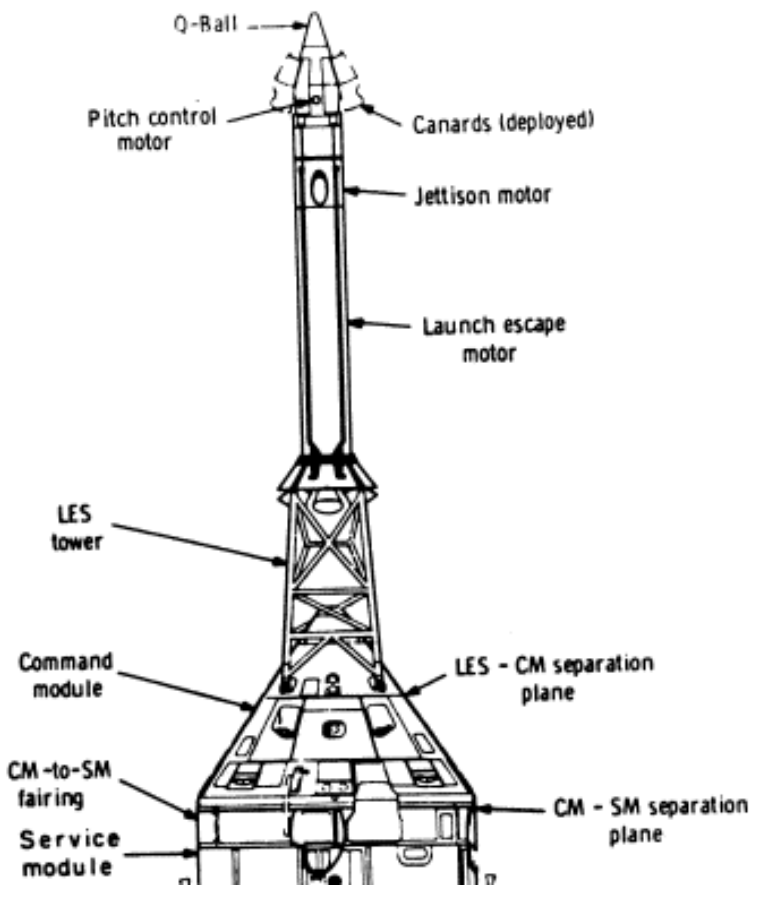

Figure 4. Apollo Launch Escape System [7].

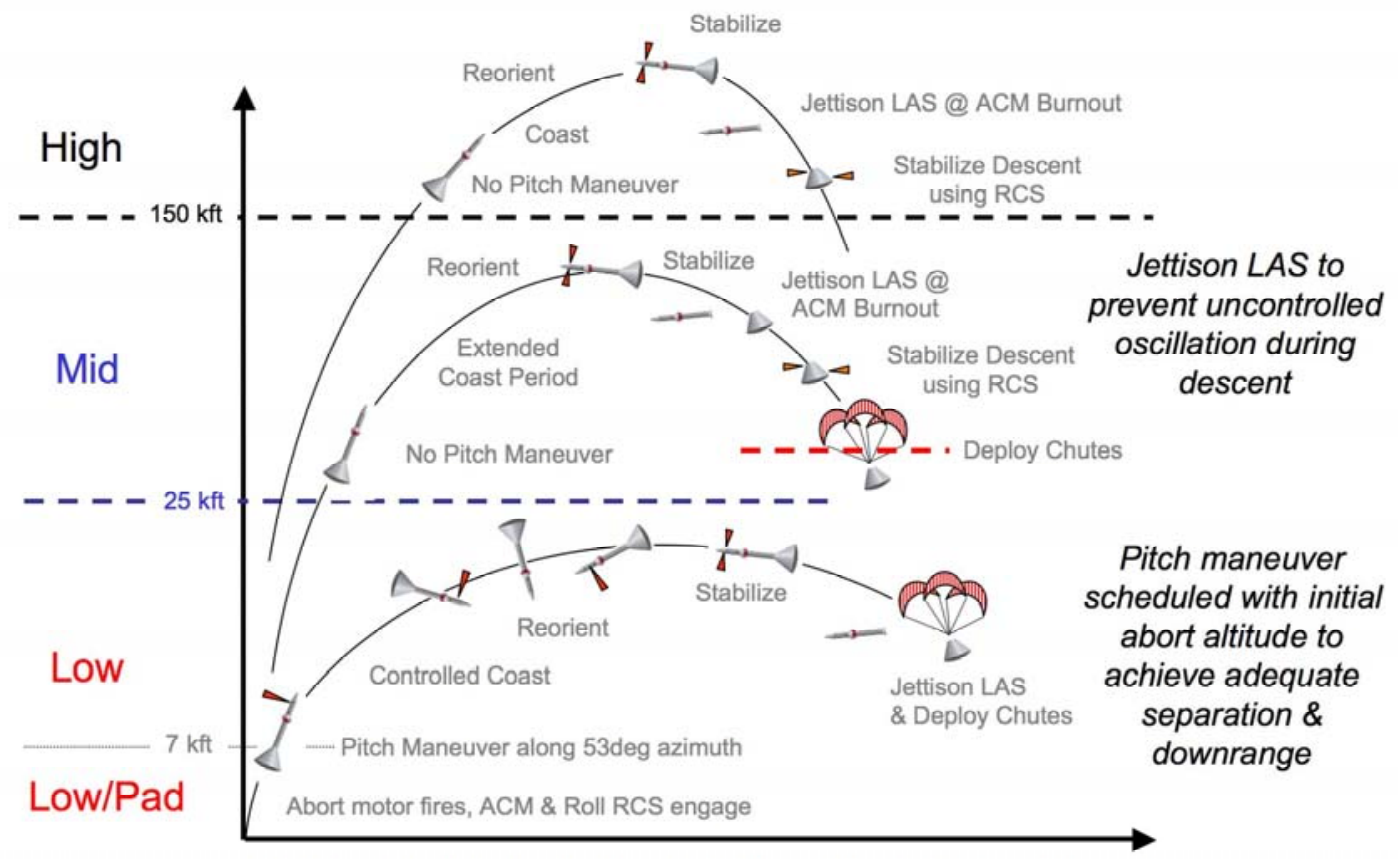

Figure 5. CEV Launch Abort System Abort Concept of Operations: Altitude Versus Time. 


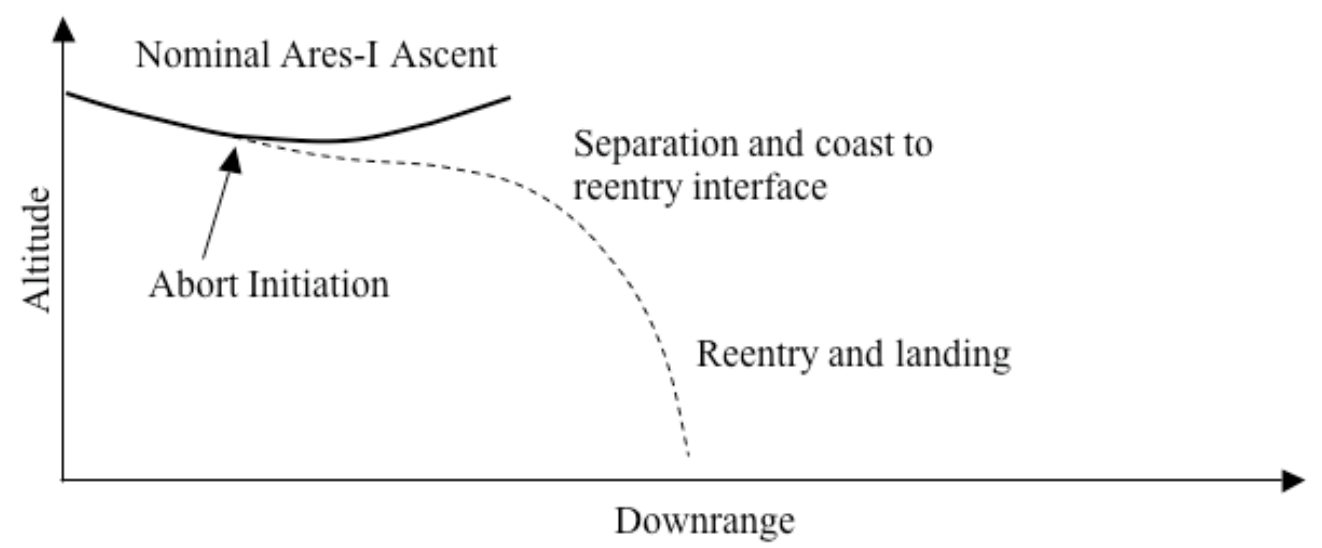

Figure 6. Untargeted Abort Splashdown: Altitude Versus Downrange Distance [8].

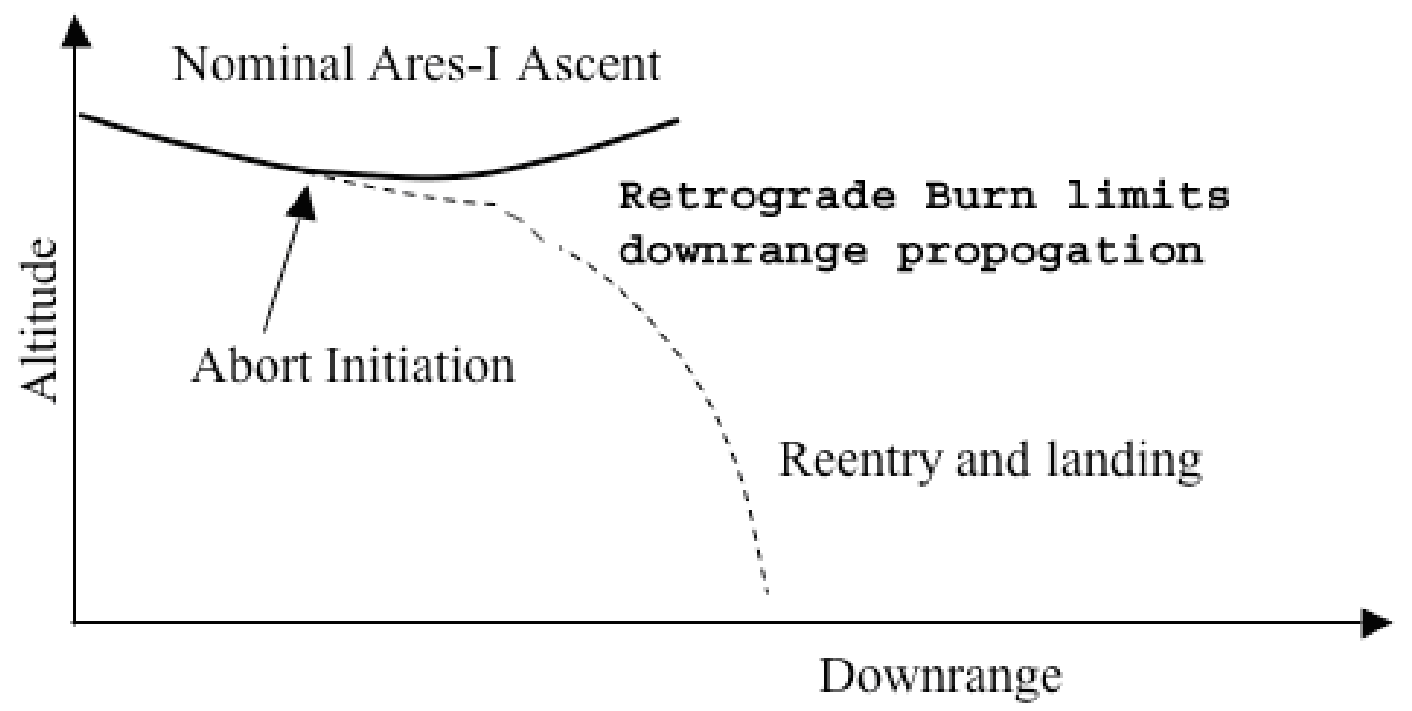

Figure 7. Retrograde Targeted Abort Landing: Altitude Versus Downrange Distance [8]. 


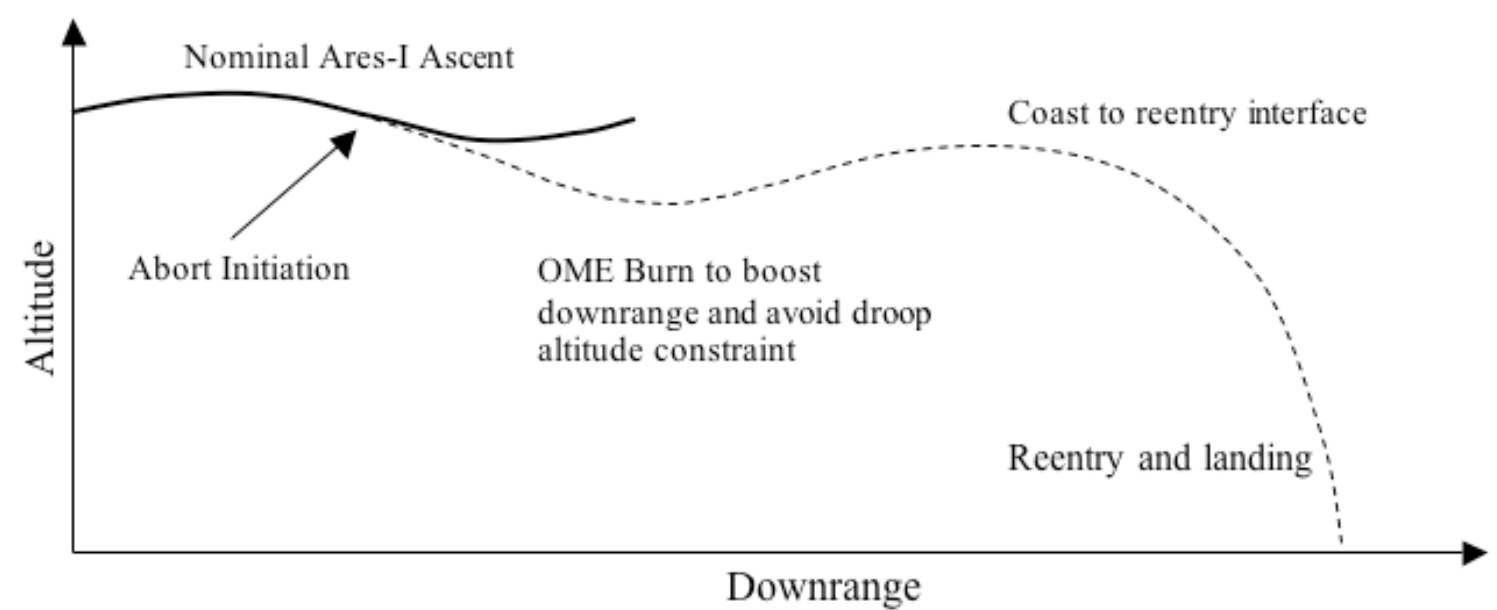

Figure 8. Targeted Abort Landing: Altitude Versus Downrange Distance [8].

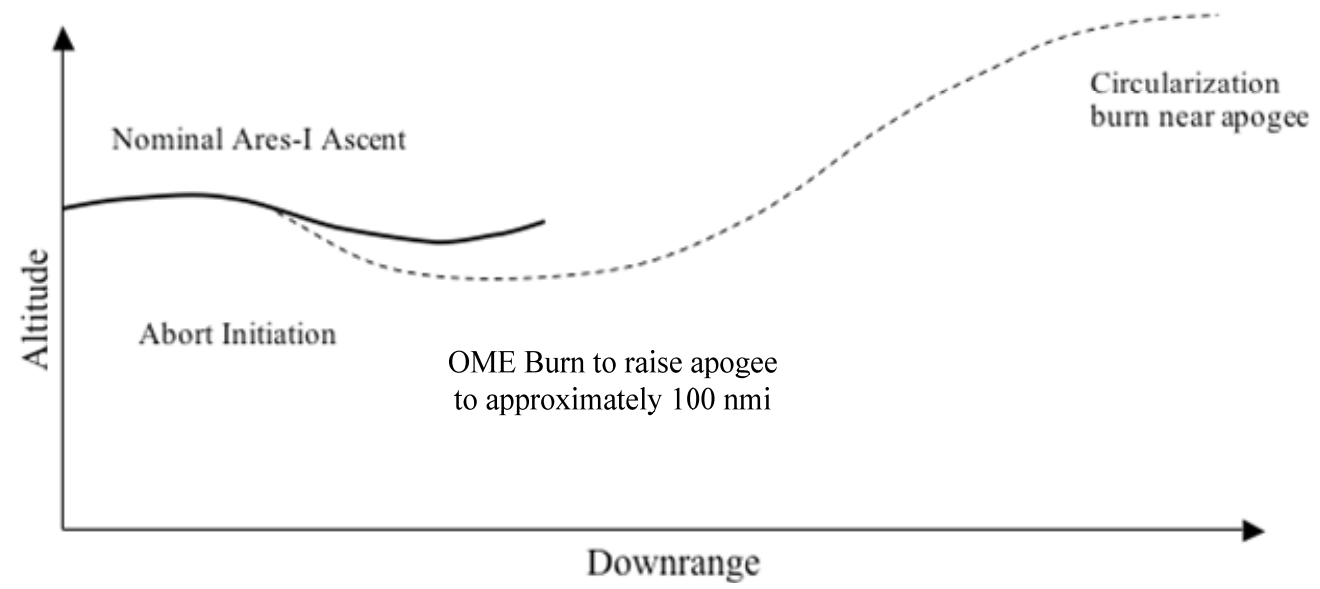

Figure 9. Abort To Orbit: Altitude Versus Downrange Distance [8]. 


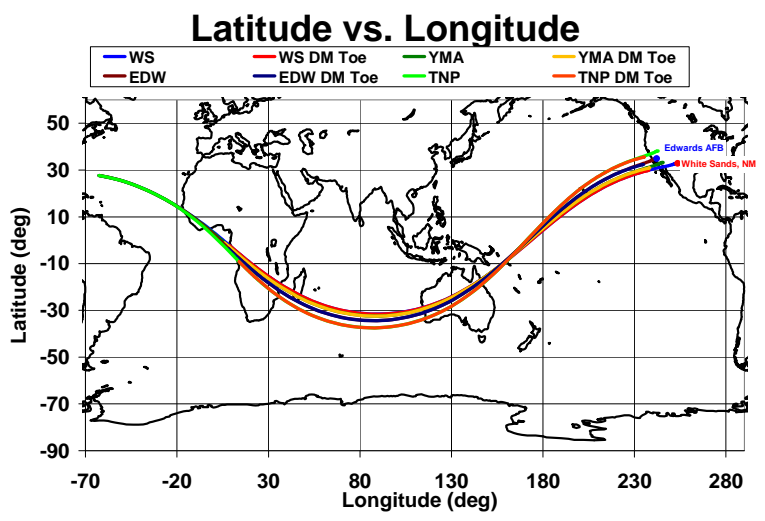

Figure 10. Abort Once Around: Lunar Ground Trace, MECO through Landing [9].

\section{Latitude vs. Longitude}

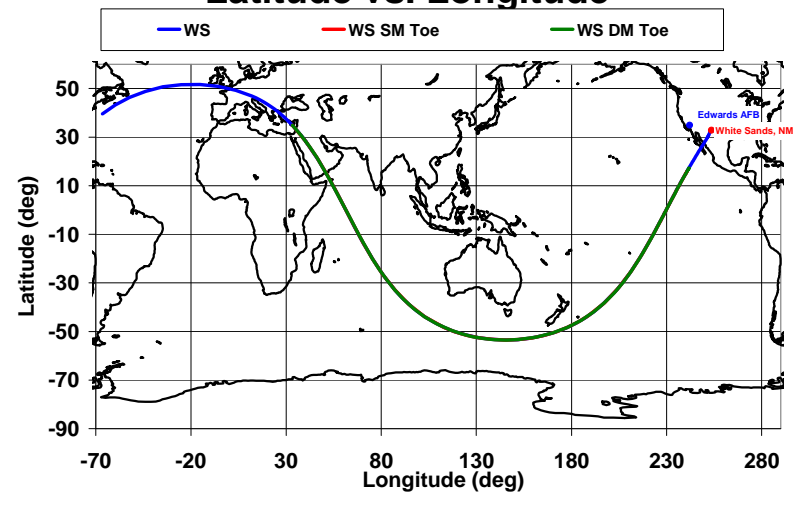

Figure 12. Abort Once Around: ISS Ground Trace, MECO through Landing [9].

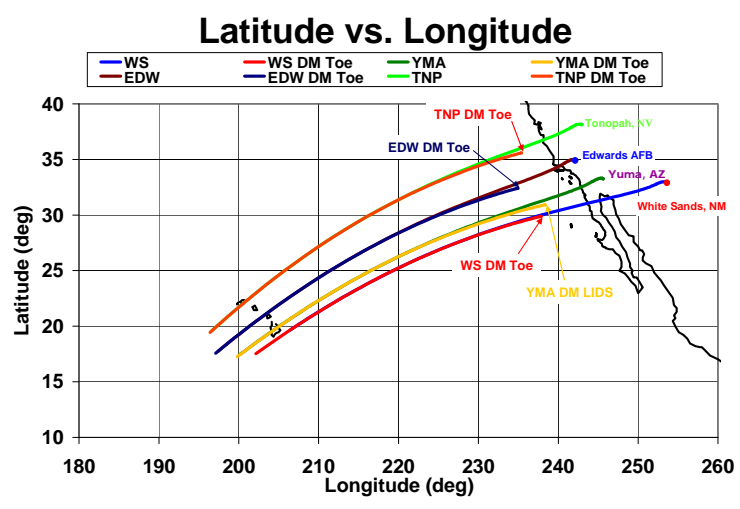

Figure 11. Abort Once Around: Lunar Ground Trace near Landing [9].

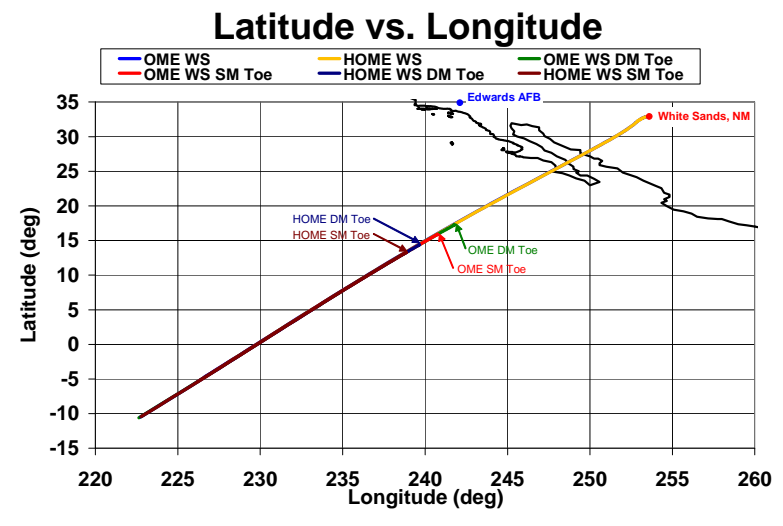

Figure 13. Abort Once Around: ISS Ground Trace near Landing [9]. 

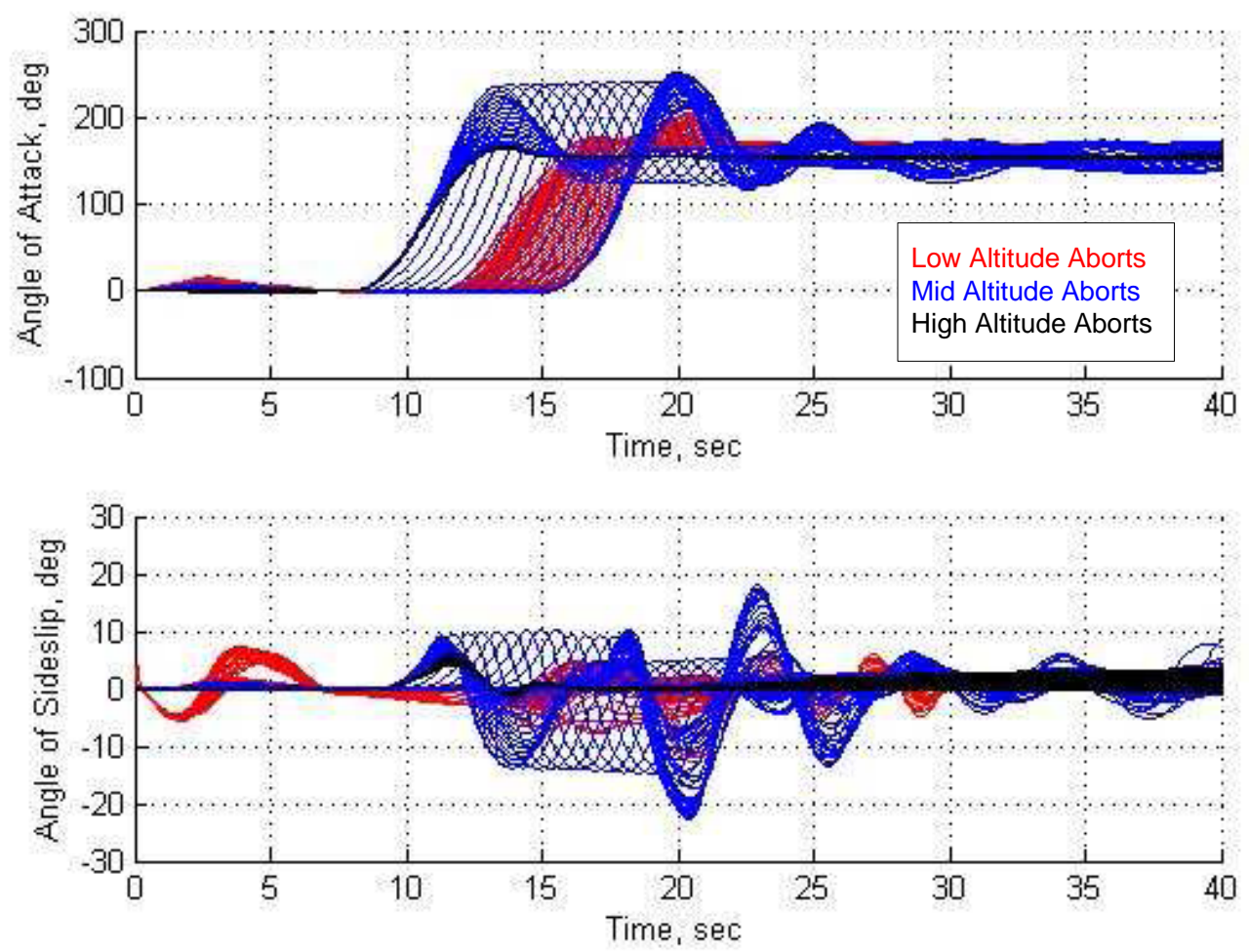

Figure 14. Angles-of-Attack and Sideslip Angle Time Histories from LAS Ascent Aborts Initiated at 1-Second Intervals along a CLV Ascent Trajectory from the Launch Pad to 300,000 feet [10].

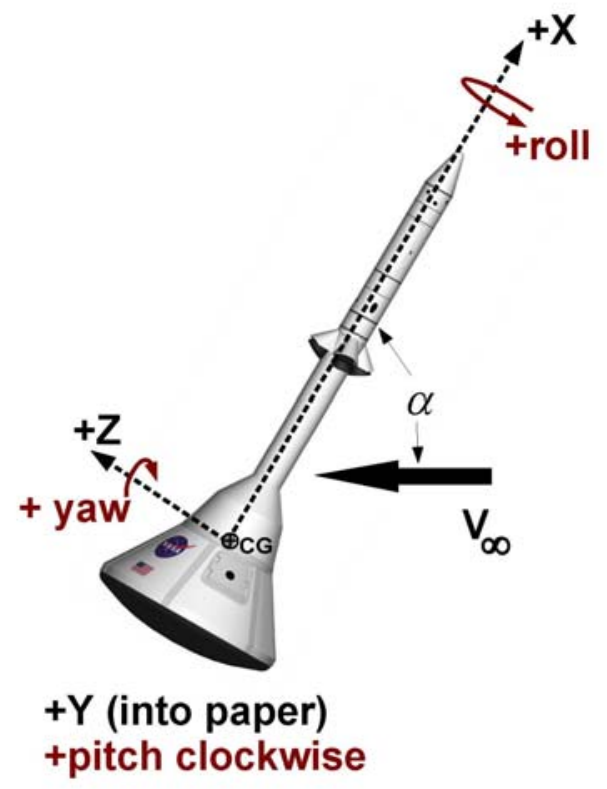

Figure 15. LAV Aerodynamics Reference System. 

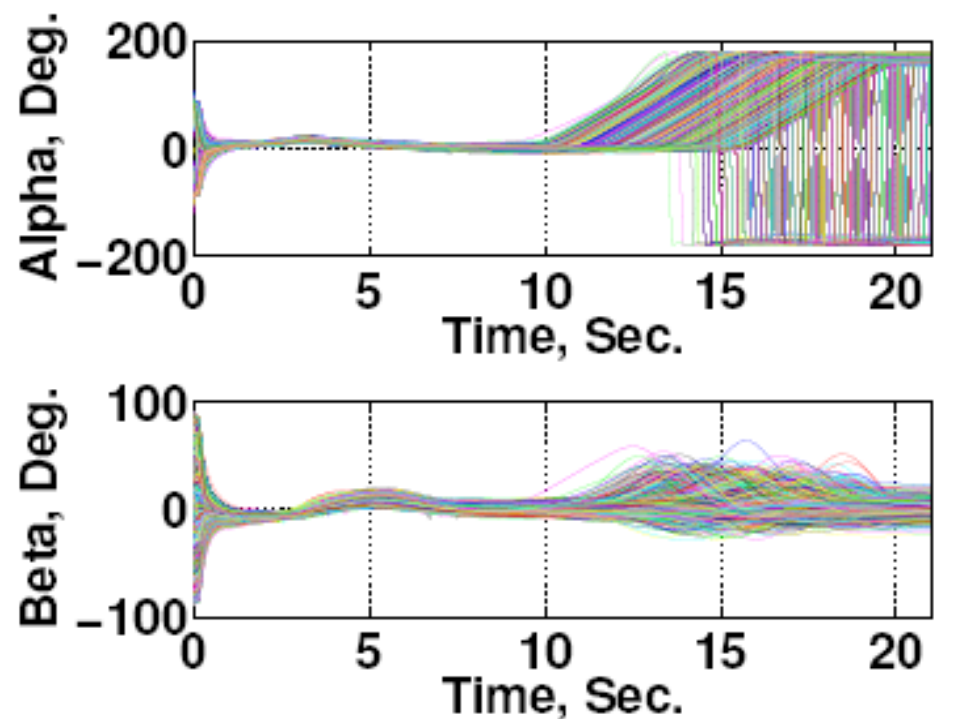

Figure 16. Angle-of-Attack and Sideslip Angle Time Histories from Monte Carlo Analysis of the Reorientation Maneuver for a Pad Abort Scenario [10]. 